\title{
Warum steigen die Prämien stärker als die Kosten?
}

\author{
Jürg Schlup \\ Dr. med., Präsident der FMH
}

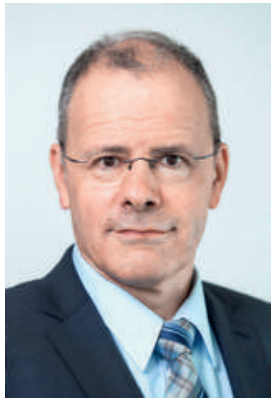

Wer in der Patientenversorgung arbeitet - und das vielleicht auch schon etwas länger - hat täglich die Gründe der steigenden Gesundheitskosten vor Augen - und findet sie wohl in der Regel eigentlich erfreulich: Unsere diagnostischen und therapeutischen Möglichkeiten nehmen stetig zu und damit auch die Zahl der Patienten, denen wir - häufig mit hochspezialisierten Leistungen - helfen können. Der medizinische Fortschritt hat die Lebensqualität und die Lebenserwartung vieler Patienten deutlich verbessert, was aber auch dazu führt, dass wir vermehrt chronisch kranke und multimorbide Patienten behandeln. Die demographisch bedingte Zunahme der über 60-Jährigen, von denen mehr als die Hälfte mindestens eine chronische Erkrankung aufweist [1], tut ihr Übriges.

\section{Seit 1996 erhöhten sich die Gesundheitskosten um $66 \%$, die Prämien jedoch um 102\%.}

Gleichzeitig bewegt sich unsere Gesundheitsversorgung im internationalen Vergleich auf einem hohen Niveau. Kaum ein Land in Europa kann einen so guten Zugang zu medizinischen Leistungen, in der Regel ohne nennenswerte Wartezeiten, oder so gute Outcomes z.B. in Hinblick auf Überlebensraten [2] und Patientenzufriedenheit [3] aufweisen. Eine so gute Gesundheitsversorgung für eine wachsende Anzahl von Patientinnen und Patienten hat zwangsläufig ihren Preis, dies zeigt auch der Anstieg der Gesundheitskosten um 66\% seit 1996.

Warum haben sich aber die Krankenkassenprämien im gleichen Zeitraum deutlich stärker - nämlich um $102 \%$ - erhöht? Dies liegt auch daran, dass ambulante Leistungen vollständig durch die Krankenkassenprämien finanziert werden, die stationären jedoch grösstenteils über Steuern. Den Kostenanstieg im ambulanten Bereich bezahlt damit zu 100\% der Prämienzahler, vom Kostenzuwachs im stationären Bereich bezahlt er lediglich $45 \%$.

Die Devise «Ambulant vor stationär» ist damit zwar im Sinne der Politik und der Patienten und spart Gesund- heitskosten - erhöht aber die Prämien! Denn jede Behandlung, die wir heute ambulant statt wie früher stationär durchführen, verstärkt die finanzielle Belastung der Prämienzahlerinnen und Prämienzahler, weil die steuerfinanzierte Subventionierung entfällt. Gleichzeitig werden viele Eingriffe nicht nur dank verbesserter Eingriffstechnik zunehmend ambulant, sondern auch insgesamt häufiger durchgeführt, weil sie durch die älter werdende Bevölkerung mehr nachgefragt werden. Im jährlichen Prämienanstieg kombinieren sich folglich die Faktoren «Fortschritt», «Demographie» und "Ambulant vor stationär». Unzutreffend und unangemessen ist es hingegen, wenn santésuisse den niedergelassenen Ärzten aufgrund höherer Konsultationszahlen eine Mengenausweitung unterstellt [4], oder das Eintreten der Ärzteschaft für einen sachgerechten ambulanten Tarif als bedeutender Kostenfaktor dargestellt wird. Tatsache ist: Ambulante Leistungen erbracht durch praktizierende Ärzte und Spitalambulatorien machen lediglich $24 \%$ der Gesundheitsausgaben aus, aber 43\% der Prämien.

Eine einheitliche Finanzierung stationärer und ambulanter Leistungen würde den Prämienanstieg bremsen.

Was heisst dies für die Zukunft? Ein möglichst effizientes Gesundheitswesen muss den ambulanten Bereich entwickeln. Wenn «ambulant vor stationär» die Prämien übermässig steigen lässt, bietet sich als Lösung eine einheitliche Finanzierung für stationär und ambulant erbrachte Leistungen an. Diese würde zumindest den Prämienanstieg bremsen, welcher durch den Ersatz stationärer Leistungen durch ambulante verursacht wird. In Bezug auf die Gesamtkosten werden wir nicht umhinkommen, anzuerkennen, dass - auch bei Ausschöpfen aller Effizienzpotentiale - die medizinischen Möglichkeiten zunehmen und damit auch die Zahl der Patienten, die von diesen profitieren möchten. 